\title{
"A COMPLETE WOMAN, BUT ONE WHO HATES WOMEN": UN PSICÓPATA DEL TERCER GÉNERO EN A SON OF THE CIRCUS, DE JOHN IRVING.
}

\author{
Cecilia Díaz Marín, Universidad de Granada \\ Email: ceciliadiazmarin@hotmail.com
}

\begin{abstract}
Resumen: Este artículo explora las complejas circunstancias que convierten en un psicópata transexual a Rahul Rai, personaje de la novela A Son of the Circus, de John Irving. Los abusos sexuales a los que lo somete su tía y tutora en su infancia, junto con un conflicto de identidad de género mal resuelto influenciado por el modelo de tercer género autóctono de la India-lxs hijras-contribuyen al desarrollo de una sociopatía que Rahul canalizará dedicándose a asesinar prostitutas en los burdeles de Bombay y Londres. Palabras clave: Literatura de los EEUU, psicopatología, identidad de género (sexo), transgénero, temas de género.
\end{abstract}

\begin{abstract}
This article explores the complex circumstances that lead Rahul Rai, from the novel A Son of the Circus, by John Irving, to become a transsexual serial killer. A victim of sexual abuse when he was a child, and in deep conflict with his gender identity (which is strongly influenced by the Indian third sex option, the hijras), Rahul becomes a sociopath who kills prostitutes in the brothels of London and Bombay for over two decades. Keywords: United States literature, psychopathology, gender identity (sex), transgender, gender issues.
\end{abstract}

“A COMPLETE WOMAN, BUT ONE WHO HATES WOMEN": UN PSICÓPATA DEL TERCER GÉNERO EN A SON OF THE CIRCUS, DE JOHN IRVING.

Las relaciones sexuales - consentidas o no- entre chicos adolescentes y mujeres maduras es una de las temáticas más recurrentes en las novelas del estadounidense John Irving, quizás porque, tal y como el escritor reveló hace unos años, él mismo fue víctima de abusos de este tipo durante su infancia. En la obra de Irving, esta clase de vínculos tienen vitales consecuencias para el posterior desarrollo de los muchachos implicados en ellas, que van desde la idealización y fijación platónica por las mujeres maduras en el caso del adolescente Eddie O’Hare, quien se ve inmerso en una relación sexual consentida con una mujer mayor que él en A Widow for One Year, hasta el desarrollo de un trauma de 
difícil superación como el de Jack Burns, protagonista de su novela Until I Find You, que es violado en su niñez por una oronda portuguesa cincuentona.

No cabe duda, no obstante, que el personaje de Irving en el que los efectos de los abusos sexuales en la infancia adquieren un cariz más devastador que en ningún otro es el de Rahul Rai, el "transexual serial killer" indio (Irving 1995: 751) que aparece en A Son of the Circus, aunque debo admitir que resultaría simplista achacar la conducta psicopática de Rahul a los abusos a los que lo somete su tía y tutora Promila Rai cuando todavía es un niño. Sin embargo, puesto que la opinión más extendida en el mundo académico es la de que ciertos factores ambientales, en funesta conjunción con otros de naturaleza genética y bioquímica, contribuyen al desarrollo de sociopatías y — ante la obvia imposibilidad de realizar un estudio hormonal o del $\mathrm{ADN}$ de un personaje literario - en este artículo analizaré los aspectos externos que pueden conducirnos a una mejor comprensión de por qué el joven Rahul Rai que aparece en algunos capítulos de la novela llega a su madurez transformado en un transexual psicópata que asesina prostitutas en los suburbios de Bombay.

\section{"UNA MISS HAVISHAM DE BOMBAY -DOS VECES"}

El pequeño Rahul Rai y su hermano mayor Subodh son criados por su tía Promila, puesto que los padres de ambos muchachos son tan pobres que no pueden hacerse cargo de su educación. Desde muy jóvenes, los dos hermanos resultan a los ojos ajenos "sexually suspect" (Irving 1995: 119). La expresión "sospechoso sexual" resulta recurrente en la obra de Irving, y suele tener connotaciones positivas para designar a personajes que unifican lo masculino y lo femenino de una forma equilibrada en una suerte de tercer género, pese a levantar ciertas suspicacias a su alrededor por ello. Por el contrario, en esta ocasión la idea de ser "sexually suspect" cobra un sentido negativo: tanto Rahul como Sudodh enarbolan una belleza amenazante y una sexualidad ambigua y agresiva que se intuye peligrosa.

Subodh, varios años mayor que su hermano, llegará a convertirse en una estrella emergente del cine indio antes de que su prometedor destino se vea truncado al fallecer en un accidente de automóvil junto con su último amante, un actor británico. Cuando Sudodh muere, Rahul es todavía muy joven, y quedará totalmente a la merced de su peculiar tía, quien es percibida por la familia del doctor Daruwalla (protagonista de la novela) como "not only a spinster but an evil, spurned woman [...] a bitter, resentful witch. She'd be an awful mother" (Irving 1995: 163), y como "a man-hater" (1995: 164).

Se atisba que el odio de Promila hacia el género masculino surge, posiblemente, por haber sido abandonada ante el altar dos veces por el mismo hombre: es una suerte de "Miss Havisham of Bombay -times two", como la define el padre del doctor Daruwalla (Irving 1995: 118). Así pues, la relación entre la tía y el menor de sus sobrinos puede considerarse "another cautionary picture of how an ill-willed adopted parent can deform a child", tal y como Marianne Novy (2007: 111) describe la de Miss Havisham y Estella en Great Expectations. Al igual que Miss Havisham, Promila Rai se consuela de su despecho amoroso a través de su dedicación a la educación de un niño, con consecuencias aún más terribles para su protegido que las que sufre la pequeña Estella en la obra de Dickens.

Mientras que Miss Havisham busca vengarse de todo el género masculino a través de su joven y bella pupila, educándola en la ausencia total de sentimientos con el propósito de 
que cuando llegue a la edad adulta rompa el corazón del mayor número posible de hombres, la formación de Rahul por parte de su tía Promila contribuye a la creación de un psicópata que asesina mujeres (“a sexually altered killer", Irving 1995: 353). Por ello, al igual que le reprocha Estella a Miss Havisham, Rahul podría espetar a su tía que "I am what you have made me. Take all the praise, take all the blame; take all the success, take all the failure; in short, take me" (Dickens 1994: 279).

Si no la totalidad de la culpa y del fracaso de quien Rahul llega a ser, no cabe duda de que sí debe gran parte de ello a Promila. La mujer profesa por el menor de sus sobrinos un afecto enfermizo, el cual la lleva a obsesionarse con la sexualidad de éste cuando sólo es un niño, quizás asumiendo que su posición de autoridad le permitirá ejercer un control y una posesión de Rahul que no pudo ejercer sobre el hombre que la desdeñó en dos ocasiones, y fantaseando con que el muchacho podrá satisfacer sus necesidades sexuales con prontitud -sólo podemos especular acerca de la naturaleza de la relación de Promila con su sobrino mayor, Subodh, al que sólo conoceremos en la novela, muy brevemente, como adulto homosexual. Observemos a continuación algunas muestras significativas que ejemplifican la naturaleza perversa de la educación del joven a manos de Promila Rai, para después pasar a analizar en qué medida ésta contribuirá al desarrollo de los rasgos psicopáticos de la personalidad del Rahul adulto.

Por ejemplo, cuando Rahul tiene unos cuatro o cinco años y ya tiene edad para utilizar el lavabo por sí sólo, Promila lo lleva al baño de señoras y lo hace orinar sentado en su regazo, justo después de hacerlo ella, para después asir su pequeño pene hasta que consigue provocarle una diminuta erección. Más adelante, antes de que el pequeño cumpla los diez años, Promila lo hace examinar por un ginecólogo, preocupada por su falta de vello corporal, y vuelve a hacerlo revisar cuando Rahul tiene doce o trece años, inquieta por su impotencia. Tía y sobrino cuentan al médico que Rahul fue incapaz de consumar el acto sexual con una prostituta (aunque en realidad, tal meretriz no existió, sino que fue Promila quien intentó infructuosamente tener sexo con el muchacho).

Existen multitud de estudios que han evaluado el daño psicológico que presentan los adultos que fueron sometidos a abusos sexuales durante su infancia, preadolescencia o adolescencia. Por citar tan sólo unos pocos, mencionaremos los análisis de C. Henry Kempe et al. (1962), Elaine Carmen, Patricia R. Rieker y Trudy Mills (con dos ensayos publicados conjuntamente por las tres estudiosas en $1984^{28}$ ), el de Mic Hunter (1991), u otros posteriores como los de Richard B. Gardner (2001) Sushma Pandey (2007) o el de Sibnath Deb y Aparna Mukherjee (2009). El artículo de Henry Kempe fue pionero en hablar del síndrome del niño maltratado y en llamar la atención de la comunidad médica y científica hacia este tipo de abusos. Mientras que los primeros estudios realizados tenían como objeto primordial - aunque no exclusivo - a las mujeres víctimas de abusos sexuales en su niñez, libros como el de Mic Hunter contribuyeron a la ampliación de tales análisis hasta los hombres que también los habían sufrido, cuestionando el mito de que hubiera un menor porcentaje de hombres víctimas de tales abusos, y de que éstos sufrieran menos

28 En adelante, para referirme al artículo "Victims of violence and psychiatric illness" lo haré diciendo Carmen et al, mientras que citaré "Hospitalization experiences of victims of abuse" como Mills et al, siguiendo el mismo orden en los apellidos de las tres teóricas con el que fueron publicados en 1984. 
sus efectos como adultos que las mujeres. Por su parte, los libros de Pandey y de Deb y Mukherjee trasladan el estudio al caso concreto de la India, donde el abuso sexual infantil trasciende con frecuencia el ámbito de lo privado y "secreto" y está ligado a la prostitución y a la explotación sexual.

Las consecuencias psicológicas que recogen éstos y otros muchos estudios ${ }^{29}$ en la materia son catastróficas: existe un mayor riesgo de que un adulto que ha sido víctima de abusos sexuales durante su infancia desarrolle adicciones a sustancias nocivas para su organismo o enfermedades mentales tales como depresiones crónicas, trastornos de pánico, esquizofrenia o bulimia, o que incluso muestre tendencias suicidas: Carmen (1984: 200) habla de la existencia de "evidence of the profoundly self-destructive behaviors that emerge after victimization". Por otro lado, según sostienen Deb y Murkherjee (2009: 120), basándose en los estudios psiquiátricos de otros autores (cf. Groth et al 1982, y Alexander-Reid et al 1994), no existe relación alguna entre homosexualidad y los abusos sexuales sufridos en la infancia por parte de un adulto del mismo sexo.

Estudios más recientes como el de Y. Gao et al. (2010) o el de Giuseppe Craparo et al. (2013) dan un paso más y se centran en el análisis de una posible vinculación entre los abusos padecidos en la niñez y la psicopatía. Craparo (2013: 1), siguiendo los hallazgos de Hare $(1991,1993)$, define la psicopatía como "a complex personality disorder, characterising individuals with emotional deficits who lack a regard for social norms, empathy, and remorse". No existe total consenso entre los expertos acerca de las causas de la psicopatía, pero, como mencioné arriba, hoy en día se suele aceptar que los aspectos genéticos y bioquímicos del individuo no bastan para explicar el origen de una sociopatía, sino que también contribuyen al mismo algunos factores ambientales (y uno de ellos puede ser, precisamente, los abusos sexuales en la infancia).

El pionero en establecer una relación entre factores afectivos e interpersonales con psicopatía fue Hervey Cleckley (1976), si bien fue Robert Hare el que profundizó en su estudio, llegando a desarrollar el Listado de Psicopatía (Hare Psychopathy Checklist o Psychopathy Checklist - revised, PLC-R en forma abreviada), que todavía hoy se emplea, a través de sus veinte ítems, como escala de calificación de psicopatía entre la población criminal. Porter (1996: 183) también señaló que la "capacity for empathetic responding [...] is 'turned off' with repeated disillusionment of the child through physical or sexual abuse or other mistreatment [...] the child's emotion being dissociated from or unconnected with cognition and behaviour over time". Por su parte, Yu Gao llega a la conclusión de que la falta de vinculación afectiva con los padres durante los primeros tres años de vida

29 Algunos de los estudios clínicos que han establecido vínculos entre el abuso sexual en la infancia y los desórdenes mentales y adicciones son el de P. E. Mullen et al., titulado "Childhood sexual abuse and mental health in adult life" (The British Journal of Psychiatry, diciembre 1993, 163 (6) 721-732), el de A Bifulco et al., "Early sexual abuse and clinical depression in adult life" (The British Journal of Psychiatry, julio 1991, 159 (1) 115122), el de David M. Fergusson et al., "Childhood sexual abuse and psychiatric disorder in young adulthood: II. Psychiatric outcomes of childhood sexual abuse" (en Journal of the American Academy of Child \& Adolescent Psychiatry, Volumen 35, número 10, 1365 - 1374); el de Kenneth S. Kendler, "Childhood sexual abuse and adult psychiatric and substance use disorders in women. An epidemiological and Cotwin control analysis", en Arch Gen Psychiatry. 2000;57(10):953-959, por citar sólo alguno de los más significativos. 
— especialmente, con la madre, o una falta de protección paterna hacia el niño-, unida con el maltrato físico ${ }^{30}$, pueden desencadenar el desarrollo de rasgos psicopáticos en adultos.

Craparo et al. sí establecen una relación más clara entre abusos sexuales en la niñez y psicopatía. Parten en su análisis no sólo del PLC-R, sino también del TEC (Traumatic Experience Checklist), desarrollado por Nijenhuis, Van der Hart, y Kruger, que menciona específicamente como experiencia traumática los abusos sexuales. Craparo llegó a la conclusión de que de sus 22 sujetos de estudio (presos condenados a prisión por delitos de sangre), 17 de ellos habían sufrido una experiencia traumática en sus primeros años de vida. Cuatro de ellos fueron víctimas de abusos sexuales, y quince, de abandono emocional; doce, de varias experiencias traumáticas (las citadas, y/o maltratos físicos y abusos emocionales). Reconoce Craparo, no obstante, que en otros estudios como el de Nijenhuis o el llevado a cabo por Thierry H. Pham (2012) el porcentaje de sociópatas que pasaron por una experiencia traumática en su primera infancia no resulta tan destacado como en el suyo. Como podemos observar, si bien Hervey Cleckley, el PLC-R de Robert Hare o Yu Gao no mencionan específicamente los abusos sexuales en la infancia, otros teóricos como Porter o Craparo sí que trazan una correlación entre ellos y la sociopatía. Siguiendo a estos últimos autores me atreveré a atribuir, si bien parcialmente, el desarrollo de un desorden psicopático a las vivencias infantiles de Rahul Rai.

De hecho, las características emocionales y experiencias vitales (no necesariamente sexuales) que los expertos en psicopatía anteriormente citados enumeran coinciden en gran medida con las de la primera infancia de Rahul Rai, y pueden explicar su falta de empatía y los rasgos psicopáticos que presenta una vez llegado a la edad adulta. Lo único que sabemos de los progenitores de Subodh y Rahul es que su padre, desesperado ante su miseria, entrega en adopción a sus dos hijos a su hermana rica, Promila, antes de que el menor cumpla los cuatro años, mientras que la madre de ambos muchachos no es ni siquiera mencionada en la novela. Los vínculos afectivos que pudieran existir entre Rahul y sus padres se cortan en su más tierna infancia: su padre es incapaz de defenderlo; su madre, figuradamente, no existe, y el rol materno es ocupado por la trastornada Promila.

Richard Gardner (2001: 13) habla de "traición sexual" para referirse a los abusos sexuales sufridos en la infancia, puesto que esta expresión "encompasses a greater range of human experience than the more common expressions 'sexual abuse', 'incest', and 'sexual trauma”, y asegura que esta traición es más grave cuanto más cercana es la relación de la víctima con el perpetrador. En el caso de Rahul Rai, la falta de vínculos afectivos en su primera infancia, que ya de por sí se consideraría un agravante o desencadenante para el desarrollo de tendencias psicopáticas en la edad adulta, se ve enconada por la traición sexual de su único pariente vivo.

Los abusos sexuales por parte de Promila hacia su sobrino tienen otra consecuencia importantísima para comprender la personalidad adulta de Rahul, aparte de ser uno de los catalizadores de cualesquiera rasgos psicopáticos genéticos (si admitimos que tal cosa existe) con los que éste pudiera estar biológicamente dotado. La historia de Rahul presenta "ele-

30 Gao emplea la palabra abuse, que en inglés puede designar tanto a los abusos sexuales como al maltrato físico; entiendo que ella utiliza esta última acepción, más amplia, por no hacer referencia alguna a los aspectos sexuales del maltrato en su estudio. 
ments common to many sexually abused men's stories: dissociation, isolation, flashbacks, sexual dysfunction, secrecy, shame, emotional and behavioral constriction, fierce rage, fear of being unmanly, and horror of being turned into a homosexual" (Gardner 2001: 13). Susan Bordo (2000: 57) incide en la vergüenza que experimenta el niño abusado, que le lleva no solo al silencio acerca de los abusos, sino también a entrar en un conflicto con su propia masculinidad: "Boys are ashamed to tell others when they are injured; the simple act of telling is an admission that they are not bearing their pain silently, stoically, "like a man"". Hablar del abuso implica, para el niño, una feminización intolerable que sumar al trauma de la violación, por lo que es frecuente que callen ${ }^{31}$. Sin embargo, el silencio de Rahul acerca de los abusos no implica su deseo de acercarse a un ideal masculino de fortaleza y estoicismo; por el contrario, encierra un anhelo de huir de su masculinidad para abrazar una feminidad ideal que lo libere de la humillación de la impotencia.

Además, la significación de la impotencia de Rahul durante los abusos de su tía Promila - que no lo son menos por su falta de consumación —, rebasa la que podría tener para un muchacho criado en el mundo occidental, puesto que la impotencia es en la India el sine qua non de la condición de hijra, ese "tercer sexo" - cuya existencia es tan aceptada como marginalizada - que se da en este país asiático. A partir de esta conexión con un colectivo que le resulta tan atrayente como repugnante, Rahul desarrollará una fijación hacia lxs hijras que, en mi opinión, se convertirá en el otro gran factor ambiental (junto con los abusos sexuales tempranos) que lo llevará a convertirse en un psicópata.

\section{"NO ESTÁ SIENDO UNO DE LOS NUESTROS 32 "}

Lxs hijras, tal y como son descritxs en "Hijras: An Alternative Sex and Gender Role in India", de Serena Nanda (1996: 373-417) y por el propio John Irving (1995: 89-91), no son exactamente una casta dentro de la sociedad india, pero sí que constituyen un grupo social muy definido, con roles establecidos tales como el de la guru o maestra y el de la chela o discípula, con comunidades propias lideradas por un consejo de ancianas con poder de decisión sobre los miembros del grupo, y con unas determinadas funciones sociales: principalmente, la bendición y animación en las ceremonias de boda o de celebración de un nacimiento de un hijo varón, y, cada vez más frecuentemente, la de la prostitución -teóricamente incompatible con el papel de un hijra, pero imprescindible por falta de otros ingresos procedentes de sus ocupaciones tradicionales.

Típicamente, lxs futurxs hijras provienen de una serie finita de situaciones personales antes de pasar a formar parte de este tercer género. Dos de ellxs, las más minoritarias, tiene un origen biológico, y son lo que se ha considerado tradicionalmente hijras de naci-

31 Bordo (2000: 57) también explica cómo los niños víctimas de abusos suelen volcarse en su cuerpo, "in an attempt to establish a private domain in which a sense of control and self-esteem can be reestablished" a través de deportes tales como el culturismo: el autor de A Son of the Circus, John Irving, hizo lo propio mediante la lucha libre.

32 "He's not being one of us" (Irving 1995: 311) dice un hijra prostitutx acerca de Rahul. La traducción es mía, y he optado por mantener el gerundio de la traducción al inglés por cuanto implica un proceso transitorio, ajustándose perfectamente a la volatilidad de Rahul Rai. 
miento $^{33}$. En la mayoría de los casos, no obstante, un futurx hijra es primero un zenana ${ }^{34}$ : un hombre, transexual o no, con sus genitales intactos, pero que en la India se considera impotente por carecer de deseo sexual hacia las mujeres, y porque al mantener relaciones sexuales con otros hombres adopta un rol pasivo: "while ambiguous male genitalia serve as the most important culturally defined sign of the hijra, in practical terms any indication of a loss of masculinity, whether impotence, effeminate behaviour or a desire for sexual relations with men in the receptor role, may be taken as a sign that one should join the hijras" (Nanda 1996: 381).

En cualquiera de estos casos, en la India se considera que lxs hijras son personas incompletas, ni hombres ni mujeres, porque carecen de la capacidad reproductiva de entrambos sexos (que es la que los determina en último grado). Es por ello que el rasgo que se considera auténticamente definitorio de un zenana no es el del establecimiento de una relación homosexual con otro hombre, sino el de presentar impotencia sexual para con las mujeres. Serena Nanda (1993: 543) señala cómo en inglés la palabra "hijra" suele traducirse cómo "eunuco" o "hermafrodita" y señala que "Impotence is the force behind both the words eunuch and hermaphrodite as they are used in India, and impotence is central to the definition of the hijra as not man". De hecho, Nanda (1993: 543) recoge cómo en el siglo XIX, un zenana que deseara ser emasculado para convertirse en hijra tenía que dormir cuatro noches con una prostituta para demostrar fehacientemente su impotencia.

Para convertirse en hijras, los zenanas deben someterse a una operación de castración, al que no consideran como una pérdida, sino como un rito de pasaje que, por el contrario, les reportará el renacimiento como un nuevo ser, un hijra. En palabras de Nanda (1996: 383), "This operation transforms an impotent man, a "useless creature", into a powerful person, a hijra, who now becomes a vehicle of the power of the Mother Goddess to bless and to curse". Desprovisto de poder sexual (im-potente), el zenana que se convierte en hijra renace revestido de un poder casi místico que es, a un tiempo, el de la diosa madre y el del ascetismo ${ }^{35}$; en otras palabras, el emasculadx es ahora "potente" en un sentido mucho más amplio que el meramente sexual.

Puesto que los aspectos principales del proceso de transformación en hijra son consabidos en la India, no ha de extrañarnos que Rahul Rai sea consciente desde muy joven de las implicaciones sociales que conlleva su imposibilidad de mantener relaciones sexuales con su tía, y que las relacione con los conflictos que tiene con su propia masculinidad. El hecho de que Promila haga examinar a Rahul por un médico a tan temprana edad también contribuye a que el muchacho desarrolle una percepción de sí mismo como alguien diferente y anormal, junto con una cierta identificación con lxs hijras -acentuada, indudablemente, por el deficiente diagnóstico del mediocre ginecólogo que lo revisa, quien asegura categóricamente que el muchacho padece "eunucodismo" sin plantearse si podía tratarse,

33 Por un lado, lxs hijras purxs son los hermafroditas, aquellxs que tienen los órganos sexuales atrofiados y que no son considerados ni hombres ni mujeres desde un punto de vista estrictamente fisiológico. Otro tipo de "hijras de nacimiento" son las mujeres que no menstrúan, cuya imposibilidad de concebir hijos les priva de la consideración social como auténticas féminas.

34 Literalmente, zenana, que proviene del persa, significa "femenino" o "propio de las mujeres" en hindi.

35 Según Nanda (1996: 375), lo ascético está en contraposición con lo erótico, dentro de las dicotomías que conforman el pensamiento hinduista, junto con las de masculino/femenino, caliente/frío. 
simplemente, de un desarrollo puberal tardío. Aún en el caso de que Rahul en realidad padeciera este trastorno ${ }^{36}$, coincido con el doctor Daruwalla en que "this diagnosis would have come at an impressionable age" (Irving 1995: 568), especialmente cuando escuchara el nombre con el que se le conocía a su supuesta "dolencia".

Una masculinidad incómoda, junto con una supuesta impotencia y un diagnóstico médico que prácticamente lo clasifica como eunuco, parecen encaminar a Rahul hacia un futuro como hijra, el modelo autóctono de tercer sexo que le resultaría más fácil de seguir para intentar pacificar sus conflictos internos. No obstante, la situación de Rahul es bastante más compleja.

Por un lado, como recoge Nanda (1996: 409), lxs hijras suelen prevenir de las castas más bajas de la sociedad india:

Most hijras appear to be drawn from the lower, though not unclean, castes. I never met a hijra who claimed to come from a Brahman family, and the taint of disreputableness that attaches to them suggests that this is not an attractive option for upper-caste persons. When a person joins the hijras, however, all former caste identities are disregarded.

Rahul, a pesar de proceder de un núcleo familiar muy pobre - o quizás precisamente por ello-, no está dispuesto a convertirse en hijra, con todo lo que ello conlleva: la renuncia a los privilegios de casta y de situación económica que adquirió al ser adoptado por su tía Promila, para pasar a tener que convivir con un grupo de hijras, y convertirse así en objeto de desprecio y recelo por parte del resto de la sociedad.

Por otro lado, Rahul, a diferencia de la mayoría de los muchachos impotentes que decidieron convirtirse en hijras en los años 70, está en contacto con el mundo occidental y cuenta con la posibilidad económica de viajar al extranjero para someterse a una operación de cambio de sexo ${ }^{37}$. Además, mientras que "most hijras were exactly what they wanted to be -they had no use for a fully fashioned vagina" (Irving 1995: 579), el anhelo de Rahul no es el de convertirse en hijra, sino el de llegar a ser una mujer completa, creyendo que de esta manera por fin se sentirá completo y en paz.

Según Susan Bordo (2000: 37), tal aspiración es común entre personas cuyo sexo biológico y su género deseado no coinciden: "Many transgendered individuals too have viewed getting rid of or acquiring a penis as the fulcrum of their search for a body that will fit their gender identity". Los genitales son considerados como la quintaesencia del sexo, y se suele articular la relación entre identidad de género y cuerpo “in terms of genital fit or

\footnotetext{
36 El "hipogonadismo hipogonadotrópico", conocido anteriormente como "eunucodismo", consiste en que "los testículos en los hombres y los ovarios en las mujeres producen pocas o ninguna hormona sexual", según el artículo homónimo de B. Wisse que se puede consultar en la web de Medline Plus.

37 Las operaciones de cambio de sexo fueron legalizadas en 1967 en el Reino Unido y en 1972 en los Estados Unidos, según recogen Gupta y Murarka (2009). En la India, sin embargo, existe un vacío legal al respecto: el cambio de sexo "tradicional" era la emasculación llevada a cabo entre lxs hijras, mientras que la opción de someterse a una intervención quirúrgica occidentalizada era un privilegio para las élites que, como Rahul Rai, podían permitirse viajar al extranjero. Sin embargo, a partir del mediático caso de Ajay Mafatlal, en 2003, las operaciones de cambio de sexo se han generalizado (Mishra, 2015), hasta tal punto que se ha revertido la tendencia y ahora son los transgénero occidentales los que acuden a la India para cambiar de sexo, por ser mucho más económico que en sus países de origen (Banerji, 2016).
} 
mismatch" (Bordo 2000: 37). Según esta idea tan generalizada, en el caso de nacer con los genitales "incorrectos", se presupone que la transformación de éstos conllevará un cambio de género que traerá la paz interior al individuo que sufría tal "anomalía", aunque nada es tan sencillo como parece, como Rahul comprobará:

That, moreover, some transsexuals seek hormonal and surgical intervention in order, by their own accounts, to realize their full humanity as male or as female implies the magnitude with which we all experience sex and gender as indispensable to our conceptions of ourselves as persons. This is not to say that human identity is reducible to sex/gender; it is to say that insofar as every aspect of that identity makes some reference implicitly or otherwise to one or both, sex and/or gender is essential to the private and public experience of human being. Indeed, no one's experience makes this clearer than the transsexual's (Wendy Lynne Lee 2010: 18).

El joven Rahul opta por realizar su cambio de género siguiendo un modelo foráneo -a través de la operación de cambio de sexo a la que se somete en Londres - frente al autóctono, el de la emasculación para convertirse en hijra, a los que en el fondo desprecia ("in the company of hijras or zenanas, Rahul had felt both different and superior", 354). De esta manera, Rahul se convierte en "A Complete Woman, but One Who Hates Women" - como Irving (1995: 351) titula el primer capítulo de la decimocuarta parte del libro, haciendo referencia a Rahul.

Rahul pasa una etapa intermedia como zenana en la que, cada vez más feminizado por causa de las hormonas que ingiere y preparándose para su inminente operación de cambio de sexo, ya da señas de su crueldad y de su paradójica fascinación y desprecio simultáneos por los ambientes más sórdidos de Bombay. Durante un tiempo, vive una doble vida en la que sigue asumiendo el rol de un joven de la alta sociedad protegido por su tía Promila, camuflándose por las noches tras la máscara de Pretty, falsa prostituta que sirve como cebo para atraer a hombres hacia los burdeles de travestis de Bombay en los cuales les prestarán sus servicios sexuales otros zenanas o hijras, que por cierto odian profundamente a Rahul por el trato vejatorio que éste les profesa, así como por ser menos agraciados físicamente que él.

Progresivamente, en los bajos fondos de la ciudad comienzan a circular terribles historias sobre Pretty, como la de que le gusta frecuentar burdeles femeninos sólo por el gusto de golpear con ensañamiento a las meretrices. En esta época, Rahul se estrena como asesino al eliminar a Dieter y Beth, dos jóvenes occidentales a los que conoce mediante un negocio de drogas en Goa. Resulta significativo que el primer hombre al que Rahul liquida sea también el último, mientras que Beth inaugura la que llegará a ser una larga lista de mujeres o transexuales asesinadxs. Sobre los despojos de la chica, Rahul crea la que se convertirá en su seña de identidad como psicópata: el elaborado dibujo de la cabeza de un elefante sobre el estómago de cada cadáver. Después de matarlos, Rahul parte con destino Londres para someterse allí a la ansiada intervención quirúrgica que lo transformará en mujer. A su vuelta definitiva del Reino Unido, Rahul contrae matrimonio con el anciano Mr. Dogar, quien ignora tanto la antigua identidad como el género original de su esposa -aunque, de hecho, nadie asocia a Mrs. Dogar con Rahul, el repulsivo sobrino de Promila Rai, quien por cierto ya ha fallecido para entonces.

Sin embargo, los demonios y luchas internas de Rahul no son aniquilados, ni tan siquiera aplacados, por el cambio de sexo al que se somete, puesto que "within Rahul a veritable 
war was being waged over the true identity of his sexual self, which, to his astonishment, was not appreciably clarified by the successful completion of his long-awaited sex change" (Irving 1995: 354). Por el contrario, parece que el cambio de sexo, junto con el hecho de haber cometido un homicidio por primera vez, desencadena definitivamente sus instintos asesinos, que le llevarán a matar a un número - indeterminado, pero en cualquier caso muy elevado - de prostitutas en la capital británica, y sobre todo en Bombay.

Irving reconoce la unicidad de cada tercer género, y cómo ésta en Rahul Rai está unido inextricablemente a su crueldad: "There's more than one way to be a third gender; but Rahul's uniqueness was inseparable from his viciousness, which extended even towards his fellow trasvestites" (Irving 1995: 354). De hecho, Rahul siente animadversión hacia todos los géneros: "Rahul had loathed being a man. In the company of homosexuals, he'd never felt he was one of them, either [...] He detested the all-too-womanly gestures of most hijras and zenanas" (Irving 1995: 354); "Rahul also loathed his fellow transsexuals" (1995: 356). Sin embargo, si bien su sentimiento de unicidad y soledad le conducen a sentir un odio hacia el ser humano en general, éste es especialmente acerbo hacia el género con el que soñaba pertenecer y el cual, al no colmar sus expectativas, le hace desarrollar una rabia aún mayor, quizás porque antes de convertirse en mujer imagina que

a new sexual identity could provide a lasting peace of mind [...] that being a woman would make him envy other women less, or not at all; he'd even thought that his desire to hurt and humiliate women would somehow evanesce. He was unprepared for how he would continue to hate them and desire to do them harm [...] His sex change had not enabled him to view other women as companionable human beings. And because Rahul still hated women, he knew he'd failed to become a woman at all (Irving 1995: 354-355).

Especialmente, a Rahul le irritan las prostitutas, "because of how lightly they regarded their sexual favors, and how they took for granted their sexual parts, which Rahul had been forced to acquire through perseverance and pain" (Irving 1995: 355), y hacia ellas dirige principalemente sus instintos asesinos.

Rahul "knew that he wasn't 'like' anyone" (Irving 1995: 356), y de un modo tristemente irónico, esta circunstancia será precisamente el desencadenante de su prematuro deceso. Cuando Rahul es finalmente capturado por el inspector Patel, la policía india se enfrenta con el problema de cómo obrar con un hombre que ahora es mujer porque se sometió en el extranjero a una operación que todavía en ese momento es ilegal en su país. La solución ideal para alguien único — "solitary confinement" (759) - es descartada por su elevado coste, y así pues, Rahul sigue el desdichado destino de otros transgénero en la India.

Cabe pensar que, dados ciertos avances legales en el reconocimiento de algunos derechos para lxs hijras ${ }^{38}$ en la India, y gracias a alegatos en favor de ellxs por parte de personalidades

\footnotetext{
38 Desde 1994, 1xs hijras son reconocidxs oficialmente como un tercer sexo, con derecho a señalar una E (de eunuco) en la casilla relativa al género en sus pasaportes, aunque paradójicamente deben identificarse como hombres o mujeres a la hora de votar. Igual les sucede en caso de tener que ser ingresadxs en prisión, con resultados a menudo más trágicos.
} 
tales como el escritor y abogado Talmar Rahesh ${ }^{39}$ o el juez K.S. Radhakrishnan ${ }^{40}$, pudieran existir ya prisiones específicas para hijras. Sin embargo tales prisiones exclusivas aún no existen, por lo que, al igual que a otros transgénero, a lxs hijras se les suele trasladar a

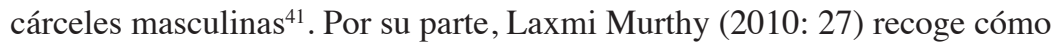

In prisons and jails, inmates are usually put into sex-segregated facilities based on their anatomy, not in their gender identity - which puts some transgender women in male facilities, greatly increasing their risk of experiencing rape and violence at the hand of guards and other inmates [...] Whether hijras should be put into men's or women's jails, continues to be a contentious issue.

Al ser anatómicamente una mujer, se descarta encarcelar a Rahul/ Mrs. Dogar en una cárcel masculina, para así protegerla de las agresiones sexuales de los demás presos. No obstante, el encarcelamiento en una prisión femenina tampoco es satisfactorio, ya que el historial homicida y la fortaleza física del antiguo Rahul conllevan un gran peligro para las presas. El inspector Patel intuye que, finalmente, la pena de muerte, elegida meramente por ser el único recurso factible para salir de este atolladero legal, pueda ser la condena aplicada a Mrs. Dogar. Finalmente, es trasladada a una cárcel para mujeres, y asesinada a golpes por los guardias como castigo por haber arrancado de un bocado la nariz a una prisionera. Como observamos, hasta el final de su vida, el conflicto entre lo masculino y lo femenino marcan el trágico destino de Rahul/ Mrs. Dogar.

Podemos afirmar, por tanto, que las tensiones, soledad y frustraciones que la pertenencia a un tercer género provocan en Rahul, unidas a y/o desencadenadas por los abusos sexuales a los que lo sometió su tía en su pubertad, desembocan en una sociopatía que encauza hacia aquellas a las que más envidia y desprecia, las prostitutas. Irving, que a través del conjunto de su obra intenta construir un tercer género ideal y equilibrado, consigue con el personaje de Rahul Rai acaso su creación más perturbadora, muestra de las terribles consecuencias que una lucha interna mal resuelta entre lo femenino y lo masculino pueden originar.

\section{REFERENCIAS BIBLIOGRÁFICAS}

BANERJI, A. 2016. "Sex-change surgery: India's new line in budget medical tourism”. 1 Jul. 2016. http://www.gmanetwork.com/news/story/565244/lifestyle/travel/ sex-change-surgery-india-s-new-line-in-budget-medical-tourism.

BORDO, S. 2000. The Male Body: A New Look at Men in Public and in Private. New York: Farrar, Straus y Giroux.

CARMEN, E.H., P. P. RIEKER, y T. MILLS. 1984. "Victims of violence and psychiatric illness". The American Journal of Psychiatry 141, 3: 378-383.

39 Acerca del encarcelamiento de 1xs hijras, Rahesh (1999: 57) señala que "In respect of hijras too a valid case can be made out that they ought not to be housed together with other male jail inmates as they be insulted, humiliated and even sexually assaulted".

40 Cf. Pandey 2014.

41 Según menciona Jaspal Singh en su artículo acerca de Seven Steps Around the Fire, una obra de teatro de Mahesh Dattani que explora la complejidad y paradojas de un caso semejante. 
CLECKLEY, H. 1976. The Mask of Sanity. Saint Louis: Mosby.

CRAPARO, G., A. SCHIMMENTI y V. CARETTI. 2013. "Traumatic experiences in childhood and psychopathy: a study on a sample of violent offenders from Italy". European Journal of Psychotraumatology 4: 1-6.

DEB, S, y A. MUKHERJEE. 2009. Impact of Sexual Abuse on Mental Health of Children. New Delhi: Concept Publishing Company.

Dickens, C. 1994 [1861]. Great Expectations. London: Penguin Books.

GAO, Y., A. RAINE, F. CHAN, P.H. VENABLES y S.A. MEDNICK. 2010. "Early maternal and paternal bonding, childhood physical abuse and adult psychopathic personality". Psychological Medicine 40, 6: 1007-1016.

GARDNER, R. B. 2001. Betrayed as Boys: Psychodynamic Treatment of Sexually Abused Men. New York: Guilford Press.

GROTH, A.N., W.F. HOBSON, y T.S. GARY, 1982. "The child molester: Clinical observations". Journal of Social Work and Human Sexuality 1, 129-144.

GUPTA, R., y MURARKA, A. 2009. “Treating transsexuals in India: History, prerequisites for surgery and legal issues". Indian Journal of Plastic Surgery : Official Publication of the Association of Plastic Surgeons of India 42(2), 226-233. 1 Jul. 2016. http://www.ncbi.nlm.nih.gov/pmc/articles/PMC2845370.

HARE, R. D. 1991. The Hare Psychopathy Checklist - Revised. Toronto: Multi-Health Systems.

1993. Without conscience: The disturbing world of the psychopaths among us. New York: Guilford Press.

HERDT, G., ED. 1996. Third Sex. Third Gender. Beyond Sexual Dimorphism in Culture and History. New York: Zone Books.

HUNTER, M. 1991. Abused Boys: The Neglected Victims of Sexual Abuse. New York: Fawcett Columbine.

IFANSASTI, U. 2009. "Photo story: the third sex”. Getty Images. 6 Junio 2016. http://www.windsorstar.com/travel/Photo+Story+Third/2210885/story.html.

IRVING, J. 1995. A Son of the Circus. London: Black Swan.

JENNY, C., T. A. ROESLER, y K. L. POYER. 1994. “Are Children at Risk for Sexual Abuse by Homosexuals?” Pediatrics 94, 1: 41-44.

KEMPE, C. H., F.N. SILVERMAN, B.F. STEELE, W. DROEGEMULLER, y H.K. SILVER. 1962. "The battered-child syndrome". JAMA 181, 1:17-24.

LEE, W. L. 2010. "Sexual identities/human subjugations: the mercurial, the out-lawed, the commodified, and the technologies of the possible". 28 Mayo 2016. http://www. academia.edu/13053085/Sexual_Identities_Human_Subjugations_the_Mercurial_the_Out-Lawed_the_Commodified_and_the_Technologies_of the Possible. 
MILLS, T., P.P. RIEKER, y E.H. CARMEN. 1984. "Hospitalization experiences of victims of abuse". Victimology 9, 3-4: 436-449.

MISHRA, N. 2015. “Is India Opening Up to Sex Change?”. 1 Jul. 2016. http://www. thequint.com/health-fitness/2015/08/24/is-india-opening-up-to-sex-change.

MURTHY, L. 2010. "Women, Men and the Emerging Other”. Ed. K. Sharma. Missing: Half the Story. Journalism as if Gender Matters. New Delhi: Zubaan. 23-54.

NANDA, S. 1993. "Hijras as neither man nor woman". The Lesbian and Gay Studies Reader. Ed. H. Abelove. London/ New York: Routledge. 542-552.

1996. "Hijras: an alternative sex and gender role in India". Third Sex. Third Gender. Beyond Sexual Dimorphism in Culture and History. Ed. G. Herdt. New York: Zone Books. 373-417.

NIJENHUIS, E. R. S., O. VAN DER HART, y K. KRUGER. 2002. “The psychometric characteristics of the traumatic experiences questionnaire (TEC): First findings among psychiatric out patients". Clinical Psychology and Psychotherapy 9: 200-210.

NOVY, M. 2007. Reading Adoption: Family and Difference in Fiction and Drama. Ann Habor: University of Michigan Press.

PANDEY, S. 2007. Psychological Consequences Of Child Abuse. New Delhi: Concept Publishing Company.

PANDEY, G. 2014. "India court recognises transgender people as third gender". 1 Jul. 2016. http://www.bbc.com/news/world-asia-india-27031180.

PHAM H. T. 2012. "Psychopathy and traumatic stress". Journal of Personality Disorders 26: 213-225.

PORTER, S. 1996. "Without conscience or without active conscience? The etiology of psychopathy revisited". Aggression and Violent Behavior 1: 179-189.

RAHESH, T. 1999. “The Third Sex and Human Rights”. Delhi: Gyan Publishing House.

SINGH, J. 2012. "Mahesh Dattani's seven steps around the fire: portraying the invisible hijra minority". Researchers World III, 4(2): 105-109.

WISSE, B. "Hipogonadismo hipogonadotrópico". MEDLINE PLUS: Biblioteca Nacional de Medicina de los Estados Unidos. 6 Jun. 2016. http://www.nlm.nih.gov/ medlineplus/spanish/ency/article/000390.htm. 